\title{
Calibration, handling repeatability, and the Maximum Permissible Error of single-volume glass instruments
}

\author{
Veronika R. Meyer · Josef Pfohl $\cdot$ Burkhard Winter
}

Received: 3 June 2010/ Accepted: 29 July 2010/Published online: 13 August 2010

(C) Springer-Verlag 2010

\begin{abstract}
The influence quantities for the uncertainty of a volumetric operation with glass instruments are calibration, repeatability and temperature. In the literature, measurement uncertainty budgets can be found, which count all three quantities separately although calibration and repeatability are merged in tabulated data to the Maximum Permissible Error. We propose that this error should be handled as a rectangular distribution in order to get a standard uncertainty. For the daily use in an analytical laboratory, the combined standard uncertainty of a volumetric operation is thus calculated from the Maximum Permissible Error plus the uncertainty of the temperature influence.
\end{abstract}

Keywords Glass volumetric instruments - Maximum Permissible Error · Measurement uncertainty · Measuring flasks · Pipettes · Single-volume glass instruments · Uncertainty of volumetric operations

Papers published in this section do not necessarily reflect the opinion of the Editors, the Editorial Board and the Publisher.

A critical and constructive debate in the Discussion Forum or a Letter to the Editor is strongly encouraged!

V. R. Meyer ( $\square)$

EMPA, Swiss Federal Laboratories for Materials Science and

Technology, Lerchenfeldstrasse 5, 9014 St. Gallen, Switzerland

e-mail: veronika.meyer@empa.ch

J. Pfohl

Brand GmbH, Postfach 1155, 97861 Wertheim, Germany

e-mail: j.pfohl@brand.de

B. Winter

DIN Normenausschuss Labor, Theodor-Heuss-Allee 25,

60486 Frankfurt am Main, Germany

e-mail: winter@dechema.de

\section{Introduction}

In its Example A1 and in Appendix G, the EURACHEM/ CITAC Guide "Quantifying Uncertainty in Analytical Measurement" (in this paper abbreviated as the Guide) describes a procedure to evaluate the combined measurement uncertainty of volumetric operations [1]. There, three independent but additive influence quantities are distinguished:

(a) Calibration. Without further explanation or discussion, the Guide uses the value which is printed on a glass instrument by the manufacturer together with the nominal volume as a \pm symbol and which is tabulated in norms, as the influence quantity stemming from calibration. The Guide treats it as a contribution with triangular distribution; therefore, it needs to be converted to a standard uncertainty by division with $\sqrt{ } 6$.

(b) Repeatability, the inevitable variation when the instrument is handled (even by a trained and experienced user), stemming from meniscus reading, droplets of liquid at the neck of measuring flasks or delivery time in the case of pipettes. A repeatability is a standard deviation by definition.

(c) Temperature effect, the influence of the difference $\Delta T$ between calibration temperature $\left(20^{\circ} \mathrm{C}\right)$ and laboratory temperature on the volume expansion of the liquid. The guide uses the expansion coefficient as a tabulated value without uncertainty, whereas a rectangular distribution is recommended for the treatment of temperature fluctuations in the laboratory. Therefore, the standard uncertainty of the temperature span is obtained by division with $\sqrt{ } 3$. 
Because the three effects are additive, the uncertainty of a volume is then calculated with the additive Gauss law of uncertainty propagation:

$$
u(V)=\sqrt{\left(u\left(V_{\text {cal }}\right)\right)^{2}+\left(u\left(V_{\text {rep }}\right)\right)^{2}+\left(u\left(V_{\text {temp }}\right)\right)^{2}}
$$

As an illustration to this approach, the treatment of a $100-\mathrm{mL}$ volumetric flask as presented in Example A1 of the Guide can be summarized as follows.

The manufacturer has printed the values $100 \mathrm{~mL} \pm$ $0.1 \mathrm{~mL}$ onto the flask. Therefore, the Guide claims that the calibration uncertainty (a) is $0.1 / \sqrt{ } 6 \mathrm{~mL}=0.04 \mathrm{~mL}$. The repeatability (b) was determined experimentally and gave a standard deviation of $0.02 \mathrm{~mL}$. In this example, the temperature variation in the laboratory is assumed to be $\pm 4{ }^{\circ} \mathrm{C}$; divided by $\sqrt{3}$, one gets a temperature uncertainty of $2.3{ }^{\circ} \mathrm{C}$. The uncertainty $u\left(V_{\text {temp }}\right)$ of the volume expansion of liquids (c) is calculated according to:

$u\left(V_{\text {temp }}\right)=V \cdot \gamma_{\text {liquid }} \cdot u(T)$

with $V=$ nominal volume of the flask, $\gamma_{\text {liquid }}$ the cubic expansion coefficient of the liquid $\left(2.1 \times 10^{-4}{ }^{\circ} \mathrm{C}^{-1}\right.$ for water) and $u(T)$ the standard uncertainty of the temperature. Equation 2 thus gives $u\left(V_{\text {temp }}\right)=0.05 \mathrm{~mL}$. By combining the three standard uncertainties according to Eq. 1, the Guide gets:

$u(V)=\sqrt{0.04^{2}+0.02^{2}+0.05^{2}} \mathrm{~mL}=0.07 \mathrm{~mL}$

In contrast to the treatment in the Guide, norms and manufacturers combine both calibration and repeatability to the Maximum Permissible Error (MPE). The tabulated and printed-on data are MPE's, not calibration values. The approach of the Guide needs to be revised with regard to the daily use of glass volumetric instruments. We emphasize that the treatments discussed here (both the one of the Guide and the one based on the MPE) include simplifications; therefore, they are not suited for a detailed uncertainty budget as it is necessary for calibration purposes and metrological laboratories.

\section{The "Maximum Permissible Error" approach}

The glass instruments used for quantitative analyses are mainly single-volume pipettes and measuring flasks. The relevant norms are EN ISO 648:2009-01 [2] and EN ISO 1042 [3], respectively. Both norms list Maximum Permissible Errors as the only quality data to be fulfilled by manufacture and proper use, i.e. by the combination of calibration and repeatability scatters. The term "Maximum Permissible Error" (MPE) is defined in DIN 1319-1 [4] or in VIM 3 [5]: the "extreme value of measurement error, with respect to a known reference quantity value, permitted by specifications or regulations for a given measurement, measuring instrument, or measuring system". The MPE is an old concept, maybe established in some industries in the 1950s. It combines the deviations from the nominal value which may come from instrument manufacture and instrument handling (in the case of an above-mentioned "measuring instrument"). For the practical use of glass volumetric instruments, this approach means:

If the instrument is properly used at the specified temperature $\left(20\right.$ or $27^{\circ} \mathrm{C}$, whatever is noted on the device), the combined effects of volume deviations from manufacturing and handling reproducibility will and must not be larger than the MPE.

As a consequence, it is only the MPE and the temperature effect which need to be combined to yield the uncertainty of the volumetric operation. The temperature parameter is to be handled as shown above in Eq. 2, although it may be discussed whether the temperature uncertainty in the laboratory should be treated as a rectangular or a triangular distribution. The MPE is a combination of the volume accuracy obtained by the manufacturing process of the glass instrument and of the possible deviations when it is used by a professional. The uncertainty distributions of the two parameters can be characterized as follows:

(a) The manufacturers take random samples during the production of a batch of identical instruments and determine their volume in accordance with ISO/FDIS 4787 [6]. In a company like Brand (Wertheim, Germany), only pipettes and measuring flasks which deviate by no more than $1 / 3$ of the MPE (determined from the random samples of a batch) are sold as class A instruments. This process could be described by a triangular distribution, because there should be a cumulation of items near the nominal value. However, the actual distribution could be broader, e.g. between the limits of the MPE; thus, we propose a rectangular distribution as a conservative assumption.

(b) The repeatability of operation is a standard deviation by definition. Note that the value included in the MPE is valid for using the instrument with pure water. For other liquids, it may be necessary to perform an in-house determination of the repeatability.

The combination of two standard uncertainties of similar magnitude but different distribution type leads to a complicated situation because the new distribution will be of mixed shape-in this case, it could be a rectangular distribution (from the production process) with Gauss-shaped sides (from the repeatability), a function whose mathematical description is not straightforward. As with any symmetrical distribution, its standard uncertainty would be the second moment of the function. (With three or more standard uncertainties of similar magnitude, the 
combination yields a normal distribution.) But from the definition of the MPE, together with a conservative approach, we propose to handle the MPE as a rectangular distribution. As a consequence, the MPE must be divided by the square root of 3 (or multiplied by 0.6 ) in order to obtain its standard uncertainty. The result then goes into the calculation of the combined standard uncertainty of the volume, together with the temperature effect:

$u(V)=\sqrt{\left(u\left(V_{\mathrm{MPE}}\right)\right)^{2}+\left(u\left(V_{\mathrm{temp}}\right)\right)^{2}}$

A 100-mL narrow-neck volumetric flask of class A has a specified MPE of $0.100 \mathrm{~mL}$. Thus, together with a temperature fluctuation of $\pm 4{ }^{\circ} \mathrm{C}$ as in the example of the Guide, one gets:

$u(V)=\sqrt{\left(\frac{0.1}{\sqrt{3}}\right)^{2}+0.05^{2}} \mathrm{~mL}=0.08 \mathrm{~mL}$

\section{Discussion}

The MPE approach yields almost identical combined standard uncertainties than the procedure proposed in the Guide (0.08 vs. $0.07 \mathrm{~mL}$ in the case of the $100 \mathrm{~mL}$ flask), but it is simpler with only two terms; compare Eq. 4 with Eq. 1 which needs three terms. Although this fact is not the main point, the MPE approach is both correct and simpler. In addition, the Guide proposes a value of the repeatability which may be too optimistic for everyday use and for nonaqueous liquids.

It can be assumed that a calculation with Eq. 5 is very conservative. However, it is not allowed to use optimistic data without experimental verification. With a pessimistic evaluation of the measurement uncertainty of any procedure, the laboratory will not present analytical results whose uncertainties are understated.

Kadis gave a thorough discussion of the topic [7] and noted in the Conclusions: "It is essential that normal variations in manipulating and reading volumetric glassware are understood to be included in the stated tolerance, and thus no additional allowance, except the temperature effects, is needed for obtaining the total uncertainty." We fully agree with this statement but our proposed approach does not assume a triangular distribution for the tolerance data listed in the norms (as does Kadis). Maybe even a treatment as proposed with Eq. 5 is too optimistic in many cases: Wampfler and Rösslein found that the uncertainty of volumetric operations with burettes is strongly underestimated (up to a factor of 4) when the bottom-up approach of the Guide is used [8].

In cases where the measurement uncertainty of a volumetric operation needs to be known with higher accuracy, it is necessary to determine the "true" capacity and the repeatability of an instrument by in-house experiments, in an analogous way as described in Ref. [6]. In fact, this needs to be recommended if the liquid in use is not pure water but has another viscosity, density and/or surface tension (body fluids, organic solvents, liquids with surfactants etc.). For the calculation of the analytical result, it will then be the experimentally found volume capacity of the instrument which will enter the equation of the measurand. The experimental standard deviation of the volume will enter Eq. 1 as $u\left(V_{\text {cal }}\right)$ and the experimental handling repeatability as $u\left(V_{\text {rep }}\right)$, besides the calculated $u\left(V_{\text {temp }}\right)$. For the latter, the best-known thermal expansion coefficient of the liquid at the operating temperature must be used; moreover, the expansion coefficient of the glass should be considered, giving the apparent thermal expansion coefficient [9]:

$\gamma_{\text {app }}=\gamma_{\text {liquid }}-\gamma_{\text {glass }}$

to be used in Eq. 2. $\gamma_{\text {glass }}$ is as follows:

Borosilicate glass, Pyrex glass (measuring flasks, burettes): $\gamma=10 \times 10^{-6}{ }^{\circ} \mathrm{C}^{-1}$;

AR glass, soda-lime glass (pipettes): $\gamma=25 \times 10^{-6}{ }^{\circ} \mathrm{C}^{-1}$; Quartz glass: $\gamma=1.6 \times 10^{-6}{ }^{\circ} \mathrm{C}^{-1}$.

It can be an open question of how to treat the temperature span of Eq. 2. The Guide proposes a rectangular distribution which is correct if only the extreme limits of the temperature occurring in the laboratory are known or assumed. However, if the laboratory is air-conditioned (or if a water bath is thermostated) around a set value, an arcsine distribution may be a better representation of the fluctuations because the extremes will occur more often than the central value. In addition, $\Delta T$ can be usually taken as the temperature difference which will take place during the performance of an analysis, such as an hour or a day, and not as the difference between the working temperature (being it measured or estimated from the possible extremes of the temperature span in the laboratory) and the official calibration temperature of 20 or $27{ }^{\circ} \mathrm{C}$. With rare exceptions only, the results of quantitative analyses performed with pipettes and measuring flasks do not depend on the absolute working temperature.

For the expansion of a volume uncertainty to a confidence level of approximately $95 \%$, i.e. to the width of two standard deviations ( $2 \sigma$ span), the appropriate coverage factor $k$ must be selected. For a rectangular distribution, it has a fixed value of 1.65 because this distribution has well-defined ends, and the expansion to $95 \%$ of its area is described by simple geometry: see the note to Table G.1 of the document "Evaluation of measurement data-Guide to the expression of uncertainty in measurement" [10]. If the temperature distribution is also looked at as of the 
rectangular type, the combination of MPE and temperature uncertainty will lead to a rectangular distribution. If the temperature effect is assumed to follow another distribution type, and if both terms are of similar magnitude, the mathematics of expansion becomes complicated: See the remarks given in paragraph 8.3 of the Guide and the detailed mathematical description in Annex G of [10]. It will be simple to just expand any volume uncertainty with the factor 1.65 (for rectangular distributions) although the factor 2.0 (for normal distributions) would be more conservative.

It muss be stressed that the approach of the Guide as well as the one proposed here is a simplification for everyday use in analytical laboratories. It neglects a number of influence parameters such as

- The influence of handling by the users of glass instruments. Non-experienced users will work with poorer repeatability than assumed by, e.g., the MPE approach. Even experienced and careful users will handle pipettes and measuring flasks with small systematic deviations which result in different absolute volumes (an observation made at EMPA laboratories);

- the rather strong dependence of the thermal expansion coefficient of liquids from temperature;

- the influence of the density, viscosity and surface tension of different types of liquids as mentioned above;

- the influence of atmospheric pressure.

This list is probably not complete.

\section{Conclusions}

We propose to use the published MPE data (to be found in norms or printed on pipettes and measuring flasks) together with the temperature effect for the determination of the measurement uncertainty of volumetric operations with glass instruments. The calculation is to be performed with Eqs. 4 and 2 and is simple. The value obtained with Eq. 4 may be too pessimistic in many cases, especially when working with pure water, but the results will lie on the safe side. They may be realistic when more viscous aqueous solutions, organic solvents or solutions with surfactants are involved. If the use of Eq. 4 seems to be too cursory, it is necessary to perform an in-house determination of the capacity and repeatability of a certain instrument, used by a certain operator (or a limited number of operators), and used with a certain liquid. The obtained data should then enter the uncertainty calculation according to Eq. 1 .

\section{References}

1. Ellison SLR, Rosslein M, Williams A (eds) (2000) EURACHEM/ CITAC, quantifying uncertainty in analytical measurement. LGC, Teddington, 2nd edn. Free download at. http://www.measurement uncertainty.org/mu/guide/index.html

2. EN ISO 648:2009-01 (2009) Laboratory glassware-single-volume pipettes. Beuth, Berlin

3. EN ISO 1042:1999-08 (1999) Laboratory glassware-one-mark volumetric flasks. Beuth, Berlin

4. DIN 1391-1 (1995) Fundamentals of metrology-basic terminology. Beuth, Berlin

5. BIPM, IEC, IFCC, ILAC, ISO, IUPAC, IUPAP and OIML (2008) International vocabulary of metrology (VIM), 3rd edn. International bureau of weights and measures, Sèvres, 2008, free download at. http://www.bipm.org/utils/common/documents/ jcgm/JCGM_200_2008.pdf

6. ISO/FDIS 4787, Draft standard, 2010-01 (2010) Laboratory glassware-volumetric instruments-methods for testing of capacity and for use. Beuth, Berlin

7. Kadis R (2004) Talanta 64:167-173

8. Wampfler B, Rösslein M (2009) Talanta 78:113-119

9. Cutnell JD, Johnson KW (2007) Physics. Wiley, Hoboken, 7th edn. Paragraph 12.5, pp 370

10. JCGM 100:2008 (2008) Evaluation of measurement data-guide to the expression of uncertainty in measurement, joint committee for guides in metrology, free download at. http://www.bipm. org/en/publications/guides/gum 